\title{
El mundo micro en el mundo nano: importancia y desarrollo de nanomateriales para el combate de las enfermedades causadas por bacterias, protozoarios y hongos
}

\section{The micro world in the nano world: Importance and development of nanomaterials for the fight against diseases caused by bacteria, protozoa and fungi}

\author{
Felipe Padilla-Vaca,,,1 Claudia Leticia Mendoza-Macías, ${ }^{*}$ \\ Bernardo Franco, ${ }^{*}$ Fernando Anaya-Velázquez, ${ }^{*}$ Patricia Ponce-Noyola,* \\ Alberto Flores-Martínez*
}

\begin{abstract}
In this paper we give an overview of the current applications of nanomaterials and nanocomposites in fields related to human health, specifically on applications to prevent the spread of pathogenic microorganisms (bacteria, protozoa and fungi) in a hospital environment through the use of nanotechnological approaches. In addition, we make a general description of how nanoparticles and nanocomposites can be effective against microorganisms resistant to conventional antibiotics. For the evaluation of the antimicrobial activity of various nanomaterials that are intended to be introduced to the market, various methods and procedures are followed according to international standards such as: International Organization for Standardization, American Association of Textile Chemists and Colorists, American Standard and Testing of Materials, Japanese Industrial Standards, among others.
\end{abstract}

KEYWORDS: infectious diseases, antimicrobial agents, antibiotic resistance, nanoparticles, nanocomposites.

RESUMEN: En este artículo damos una revisión general de las aplicaciones actuales de nanomateriales y nanocompuestos en campos relacionados con la salud humana, específicamente sobre las aplicaciones para prevenir la propagación de microrganismos patógenos (bacterias, protozoarios y hongos) en un ambiente hospitalario mediante el uso de enfoques nanotecnológicos. Además, hacemos una descripción general de cómo las nanopartículas y los nanocompuestos pueden ser efectivos contra microrganismos resistentes a los antibióticos convencionales. Para la evaluación de la actividad antimicrobiana de diversos nanomateriales que se pretenden introducir al mercado, se siguen diversos métodos y procedimientos de acuerdo con estándares internacionales tales como: International Organization for Standardization (iso), American Association of Textile Chemists and Colorists, American Standard and Testing of Materials, Japanese Industrial Standards, entre otros.

PALABRAS CLAVE: enfermedades infecciosas, agentes antimicrobianos, resistencia a los antibióticos, nanopartículas, nanocompuestos.

* Universidad de Guanajuato, División de Ciencias Naturales y Exactas, Guanajuato, México.

1 Autor para correspondencia: (padillaf@ugto.mx). 


\section{Nanotecnología}

La nanotecnología se define como el estudio de las propiedades de la materia en la escala nanométrica (figura 1), en particular se enfoca en las propiedades dependientes del tamaño del estado sólido de los materiales (Mulvaney et al, 2015). Se habla de nanotecnología cuando se puede hacer la manipulación de las propiedades de una molécula a la vez para obtener propiedades nuevas o diferentes de los materiales en escala macro o micro. Los fenómenos naturales en la escala macro obedecen a las leyes de la mecánica clásica, mientras que, entre más pequeños son los objetos, los fenómenos cuánticos predominan en el comportamiento de los nanomateriales (figura 1).

La nanotecnología ofrece la posibilidad de crear y diseñar nanopartículas (NPs) con requisitos específicos. Esta área ha sido ampliamente explorada por la química, la física, la biología, la medicina y las ingenierías, entre otras disciplinas. La nanotecnología tiene el potencial de optimizar procesos industriales, crear productos innovadores y aportar soluciones a problemáticas críticas para la sociedad. El término NP se refiere a partículas en donde

FIGURA 1. Representación gráfica de la escala nanométrica.

Escala

Metros Ejemplos de Objetos

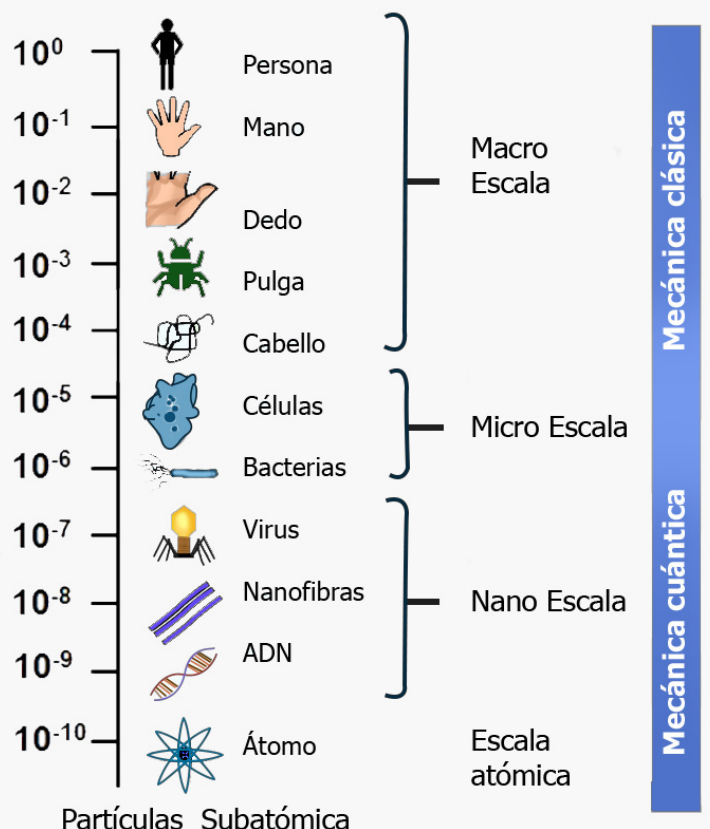

Medios de percepción
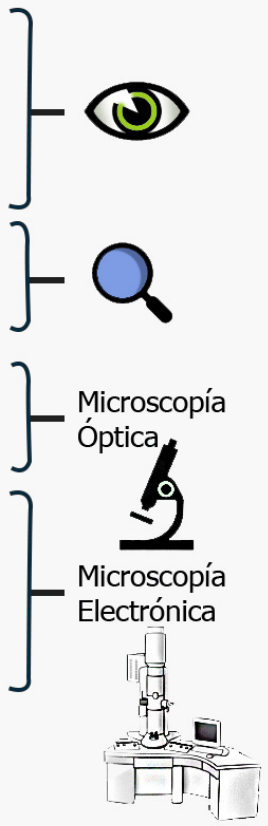

Fuente: Elaboración de los autores. 
por lo menos una de sus dimensiones se encuentra entre 1 y 100 nanómetros (Auffan et al., 2009). Recientemente, su síntesis y caracterización han sido de gran interés científico e industrial puesto que presentan un aumento exponencial de su área en comparación con su volumen, lo que repercute en la mejora de sus propiedades mecánicas, térmicas, ópticas y eléctricas, entre otras, en comparación con materiales micrométricos.

El uso de moléculas basadas en el carbono en diferentes combinaciones estructurales ha generado tubos (nanotubos), esferas (fulerenos) o superficies planas (grafeno), con constituyentes no sólo de carbono, sino también de metales, cerámicas y polímeros (Dresselhaus et al., 2010). Existe una gran variedad de NPs dentro de las cuales destacan las nanofibras, nanoarcillas, nanoalambres, entre otros; además de NPs metálicas tales como plata, oro, cobre, titanio, zinc, y sus óxidos metálicos correspondientes en escala nanométrica, en donde sus características particulares dependen principalmente de su tamaño, forma, geometría y superficie (Fedlheim y Foss, 2001). En particular, la introducción de cargas nanométricas en matrices poliméricas sintéticas o naturales ha generado nanocompuestos con características fisicoquímicas mejoradas con aplicaciones en el cuidado de la salud (EspañaSánchez et al., 2014; Luna-Hernández et al., 2017).

\section{Enfermedades infecciosas}

A pesar del hecho de que vivimos en una era de tecnologías avanzadas e innovadoras para dilucidar mecanismos de enfermedades y el diseño molecular de nuevos medicamentos, las enfermedades infecciosas siguen siendo uno de los mayores desafíos relacionados con la salud en todo el mundo. Los principales inconvenientes para los agentes antimicrobianos convencionales son el desarrollo de resistencia farmacológica múltiple y los efectos secundarios adversos. El desarrollo de antibióticos no convencionales es una alternativa para prevenir la infección y combatir los microrganismos patógenos, incluyendo bacterias, hongos y protozoarios. En particular, varias clases de NPs antimicrobianas y nanocompuestos para el suministro de antibióticos han demostrado su eficacia para prevenir y tratar enfermedades infecciosas (Ae y Young, 2011).

Uno de los sitios de mayor riesgo para contraer una infección por microrganismos es dentro de un hospital, puesto que la susceptibilidad de los pacientes se incrementa por la exposición constante a los agentes infecciosos, los cuales, generalmente, son resistentes a los antibióticos convencionales. De acuerdo con el Centro de Control y Prevención de Enfermedades de los Estados Unidos y la Organización Mundial de la Salud, una infección intrahospitalaria (IIH) se define como la infección adquirida por pacientes dentro de un recinto de atención a la salud, la cual se encuentra ajena al motivo inicial de ingreso (Horan et al., 1992). Hoy en día, muchos microrganismos son resistentes a uno o varios agentes antimicrobianos y hay estu- 
dios que indican que el uso indiscriminado de estos promueve la transferencia de la información genética permitiéndoles a las bacterias resistir a estos fármacos (Jutkina et al., 2017). Aproximadamente, entre un 5 y $10 \%$ de los pacientes que ingresan a un hospital adquieren una IIH, de los cuales el $5 \% \mathrm{fa}-$ llecen por la misma causa, ocasionada principalmente por bacterias tales como Staphylococcus aureus, Pseudomonas aeruginosa, Klebsiella pneumoniae entre otras (Melzer et al., 2003).

Las IIHs por protozoarios parásitos son relativamente pocas en comparación con los otros agentes infecciosos, afectando principalmente a pacientes inmunocomprometidos; dentro de éstos tenemos: Pneumocystis carinii, Toxoplasma gondii y Cryptosporidium spp. Sin embargo, presentan altas tasas de prevalencia y amplia distribución universal, principalmente en las regiones tropicales y subtropicales. A pesar del incremento de recursos terapéuticos eficaces y del establecimiento de programas de control, sobre todo en la población infantil (www.cdc.gov/parasites/es/about.html; Short et al., 2017). Es importante la revaluación de las pautas de tratamiento y desarrollo de nuevos fármacos debido a la creciente resistencia de los parásitos, aunque otros factores también contribuyen a este fenómeno (Ponte-Sucre et al., 2017).

Estudios epidemiológicos indican que el número de infecciones fúngicas sistémicas ha aumentado significativamente durante las últimas décadas, generando importantes problemas de salud pública por lo cual es una necesidad desarrollar agentes novedosos y eficientes contra las especies que causan las micosis (Havlickova et al., 2008). Candida albicans es un hongo que afecta al ser humano y uno de los más importantes dentro de las IIH (Melzer et al., 2003), pero también hay una gran variedad de hongos que afectan a los animales y a las plantas.

\section{Desarrollo de nuevos agentes antimicrobianos}

\section{Bacterias}

La nanotecnología se ha utilizado cada vez más para aplicaciones médicas y es de gran interés su enfoque para matar o reducir la actividad de numerosos microrganismos. En la última década, se ha comenzado a explotar el potencial tecnológico de las NPs como microbicidas o acarreadores de fármacos para que lleguen de manera rápida y eficaz al sitio blanco de acción. En los últimos 20 años se han publicado más de 5 mil artículos sobre la actividad antimicrobiana de las NPs y nanocompuestos, de los cuales más del $80 \%$ son sobre bacterias (www.ncbi.nlm.nih.gov/pubmed).

Las NPs metálicas han demostrado poseer propiedades bacteriostáticas y bactericidas asociadas con un incremento en el área de contacto, aumentando sus actividades físicas y químicas, siendo consideradas como los nuevos agentes antimicrobianos de nueva generación (Seil y Webster, 2012). La estructura física de una NP y la forma en que interactúa y penetra en las bacterias parece proporcionar mecanismos bactericidas únicos. Se han publicado 
un buen número de trabajos enfocados en determinar el mecanismo antibacteriano, empleando NPs metálicas de diferente tamaño y forma, varias especies de bacterias patógenas y no patógenas, y con diferentes enfoques experimentales, siendo las NPs de plata las más empleadas. Sin embargo, en los últimos años el uso de NPs de cobre se está incrementando por su alta actividad antimicrobiana y por su menor costo que la plata (España-Sánchez et al., 2014). Los estudios se han enfocado en analizar las etapas tempranas (España-Sánchez et al., 2017) y tardías del daño en las bacterias ocasionado por las NPs metálicas (Vimbela et al., 2017).

Las NPs metálicas inician el proceso antibacteriano a través del contacto con la pared celular bacteriana mediante interacciones electrostáticas, liberando iones que alteran la permeabilidad de la bacteria, favoreciendo la penetración de las NPs y su posterior interacción con biomoléculas y componentes intracelulares (Morones et al., 2005). Estudios recientes han demostrado que las NPs metálicas además de causar daños estructurales en la membrana celular, generan especies reactivas de oxígeno (ROS) (Wang et al., 2014) que provocan daño al material genético de los microrganismos, afectando el proceso de replicación. Los iones metálicos de las NPs interaccionan con la carga negativa de las proteínas con las que forma complejos afectando su función (Lok et al., 2006) e interfieren con la fosforilación de proteínas (Shrivastava et al., 2007). Las NPs metálicas también producen la peroxidación de lípidos dañando la integridad de la membrana celular por estrés oxidativo (Tedesco et al., 2010) (figura 2). Finalmente, el efecto antibacteriano es la sumatoria de diferentes factores, dentro de los cuales destacan la naturaleza química de las NPs, su morfología, el proceso de liberación de iones en su superficie y las diferencias en la estructura celular bacteriana.

FIGURA 2. Mecanismo de acción de las nanopartículas sobre células bacterianas.

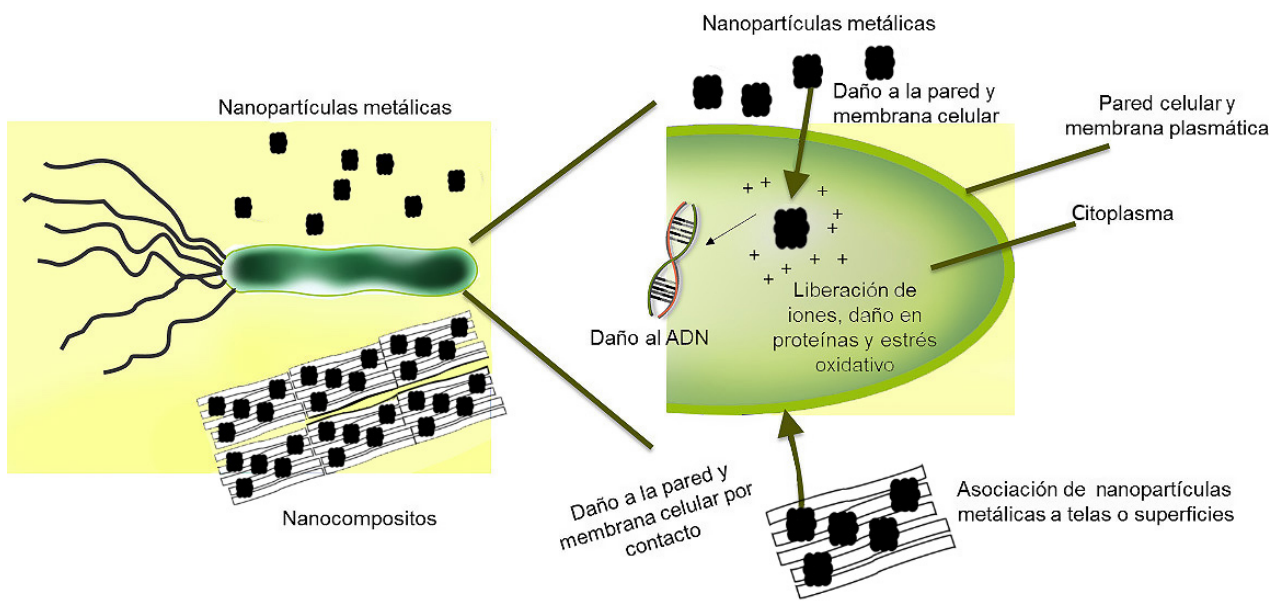

Fuente: Elaboración de los autores. 
Las NPs de $\mathrm{Cu}$ y $\mathrm{Ag}$ han mostrado gran actividad antibacteriana sobre cepas de $S$. aureus y $P$. aeruginosa sensibles o multirresistentes a antibióticos convencionales (González-Vargas et al., 2017). La incorporación de NPs metálicas en polímeros con propiedades antimicrobianas, tales como la plata y el cobre entre otras, ha permitido obtener nanocompuestos capaces de prevenir el crecimiento y la propagación de microrganismos, siendo las NPs de plata las cargas nanométricas más empleadas en polímeros sintéticos (España-Sánchez et al., 2014) y naturales (Luna-Hernández et al., 2017). Asimismo, las NPs son eficientes en la ruptura de estructuras de resistencia conocidas como biopelículas, compuestas por células muertas, ADN, proteínas y un polisacárido extracelular que incrementa la resistencia de los microrganismos a los antibióticos convencionales y a las defensas del hospedero (Kim, 2016). También se han descrito NPs que liberan fármacos de actividad antibacteriana bajo ciertos estímulos (Chiang et al., 2015).

\section{Protozoarios}

Entre la variedad de seres vivos, los protozoarios se caracterizan por ser microrganismos unicelulares eucarióticos con capacidad para sobrevivir en diferentes ambientes. Existen aquellos adaptados a vivir en condiciones aeróbicas y los que pueden hacerlo en condiciones anaeróbicas. La mayoría son de vida libre, pero algunos son comensales y otros pueden ser patógenos para el ser humano o para animales, por lo que se han diseñado NPs o nanocompuestos para su aplicación en el ámbito clínico o veterinario. Aunque el número de reportes es reducido, se han realizado diversas investigaciones sobre el uso de las NPs metálicas o nanocompuestos sobre los protozoarios (figura 3).

Se han probado NPs de plata que mostraron actividad antiamibiana en contra de trofozoítos de Acanthamoeba castellanii, responsable de la encefalitis y queratitis en el humano. Las nanopartículas de dicho elemento preparadas en un extracto de una planta, mostraron $100 \%$ de inhibición del parásito con una concentración de $50 \mu \mathrm{g} / \mathrm{ml}$ (Borase et al., 2013). Las NPs de selenio, biosintetizadas por Bacillus sp., mostraron buena actividad en contra de Leishmania major. También se han probado NPs de óxido de zinc sobre la misma especie de Leishmania, mostrando que son citotóxicas para este parásito (Akbari et al., 2017). Se ha estudiado el efecto de NPs de plata sobre Giardia en ratas infectadas con este parásito, obteniéndose buenos resultados después de evaluar el número de quistes/trofozoítos en las heces (Said et al., 2012). Por otro lado, se observó buena actividad de NPs de oro, plata o platino sobre Toxoplasma gondii (Benelli, 2018). Asimismo, se investigó el efecto de las NPs de plata u óxido de cobre sobre Entamoeba histolytica y Cryptosporidium parvum, encontrándose alta actividad inhibitoria (Saad et al., 2015).

También se han investigado los efectos de NPs de plata u oro preparadas a partir de extractos de plantas sobre Leishmania, observándose que las primeras tuvieron una IC50 de $4.37 \mu \mathrm{g} / \mathrm{ml}$ y las segundas de $5.29 \mu \mathrm{g} / \mathrm{ml}$, respectivamente (citado por Aderibigbe, 2017). 
FIGURA 3. Tipos de nanopartículas para combatir las infecciones por parásitos protozoarios.

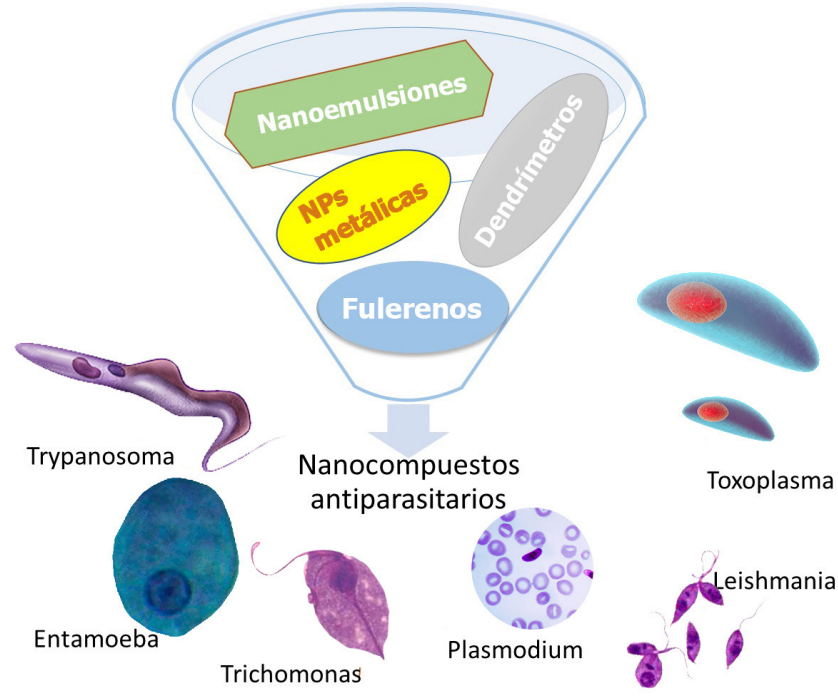

Fuente: Elaboración de los autores.

Además del efecto antiparasitario de las NPs metálicas, también se ha aplicado la nanotecnología a fármacos antiparasitarios ya descritos, desarrollando preparados en forma de NPs para evaluar su efecto sobre los parásitos. Se analizaron NPs de benznidazol sobre varios estadios de vida de Trypanosoma cruzi y sobre la evolución de la infección experimental en ratones, obteniéndose buenos resultados en ambos casos (Scalise et al., 2016). En un estudio posterior, se encontró que tales nanopartículas en un modelo similar de 30 días de evolución, se logró una inhibición del $60 \%$ a una concentración de $25 \mathrm{mg} / \mathrm{kg} /$ día (Rial et al., 2017). Con respecto a Plasmodium falciparum, las NPs de plata tuvieron mayor actividad que la cloroquina; la IC50 fue de aproximadamente $50 \mu \mathrm{g} / \mathrm{ml}$ tanto en cepas sensibles como en resistentes a dicho fármaco (citado por Aderibigbe, 2017).

Las NPs poliméricas como nanocápsulas y nanoesferas se han aplicado como sistemas acarreadores o de liberación controlada y/o dirigida de fármacos. En uno de los estudios la artemisinina se encapsuló en NPs de ácido poliláctico o poliglicólico, que fueron eficaces para eliminar los parásitos de Leishmania donovani de macrófagos de ratón in vitro, mientras que en otro enfoque el sulfato de paromomicina fue introducido en NPs de lípidos sólidos y fue efectivo en ratones infectados con Leishmania (Akbari et al., 2017). Otros nanomateriales que se han investigado sobre parásitos, han sido los nanotubos de carbonos, con multicapas, los cuales tuvieron un efecto letal sobre los trofozoítos de E. histolytica usando concentraciones de $100 \mu$ g por cada 100,000 amibas (Elias y cols., 2007). 


\section{Hongos}

En el caso de los hongos, organismos que pueden atacar a plantas o al humano, es de interés el diseño de nanomateriales que funcionen como antifúngicos o que prevengan posibles infecciones por estos microrganismos, pues son muy difíciles de eliminar cuando atacan al huésped. Uno de los hongos patógenos oportunistas del humano es la levadura del género Candida, que causa la candidiasis, siendo las especies $C$. albicans, $C$. parapsilosis y $C$. glabra$t a$ las principales causantes de esta enfermedad. Se ha descrito que bioconjugados de NPs de oro presentan alta actividad antifúngica contra C. albicans y Saccharomices cerevisiae (Das et al., 2009). NPs de plata y oro son potentes antimicóticos contra levaduras patógenas oportunistas (Candida y Cryptococcus) y dermatofitos (Microsporum y Trichophyton) a concentraciones que no resultaron tóxicas para queratinocitos en cultivo (Rónavári et al., 2018). La mezcla del fungicida Trihexad 700 WP con NPs de plata conjugadas con quitosano presentó un efecto fungicida sinérgico contra Pyricularia oryzae, el hongo que ataca al arroz (Pham et al., 2018). Las NPs de cobre también tienen efecto sobre diferentes levaduras no patógenas (S. cerevisiae) y patógenas (Candida), observándose que, al comparar la dosis letal media, los hongos patógenos fueron menos sensibles (Islas-Robles, 2013). Esta menor susceptibilidad está relacionada con la expresión de la cobretioneína que tiene la función de quelar el cobre intracelular modulando sus efectos tóxicos (Lerch, 1980). La actividad de las metalotioneínas es un mecanismo de detoxificación para diferentes metales pesados (Hamer, 1986) que podría incrementar las dosis tóxicas de NPs metálicas para levaduras.

\section{Estrategias para combatir a los microrganismos patógenos usando la nanotecnología}

Los nanocompuestos con nuevas propiedades fisicoquímicas tienen una amplia gama de aplicaciones, como son la incorporación de componentes biológicos (como las enzimas) o bien incorporando NPs metálicas con aplicaciones en la industria alimenticia y de empaquetamiento de perecederos, en la industria de textiles, en materiales quirúrgicos y recientemente con un potencial para combatir enfermedades (Wu et al., 2017). Las NPs y nanomateriales, no sólo tienen impacto como antimicrobianos, también en sistemas de liberación de fármacos, como acarreadores y adyuvantes para vacunas, recubriendo dispositivos médicos y en el diagnóstico de los patógenos (figura 4).

Las NPs se pueden funcionalizar para facilitar la administración de medicamentos antimicrobianos, superando así algunas de las limitaciones de los agentes terapéuticos. En los últimos años, la encapsulación de fármacos en los sistemas de NPs ha surgido como una alternativa innovadora y prometedora que mejora la eficacia terapéutica y minimiza los efectos secundarios indeseables de los medicamentos (Zhang et al., 2010). La síntesis verde de 
FIGURA 4. Uso de nanomateriales como antimicrobianos y en diferentes aplicaciones para el combate de las infecciones microbianas.

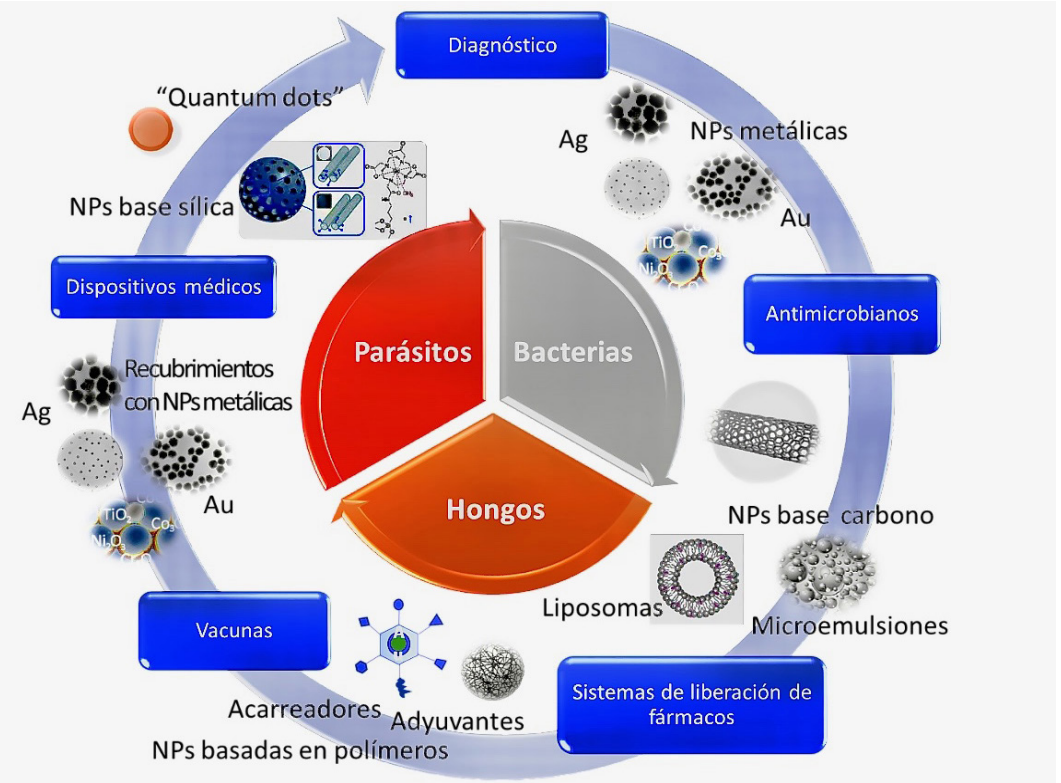

Fuente: Elaboración de los autores.

NPs metálicas y su incorporación a sistemas con fármacos ha permitido obtener efectos sinérgicos sobre los microrganismos (Pham et al., 2018).

La importancia e impacto de las enfermedades causadas por microrganismos hace imperativo desarrollar sistemas para el diagnóstico temprano y la contención de éstas. En vista de la urgencia de diagnósticos sensibles, específicos, robustos y rápidos, se han realizado numerosos avances en el área de diagnóstico. Uno de los enfoques es utilizar nanomateriales para lograr la detección de agentes infecciosos, incluso en medios complejos como la sangre y la orina. Se han abordado varias estrategias para el diagnóstico de parásitos en el laboratorio, las cuales aún se encuentran a nivel experimental y pueden extrapolarse a otros microrganismos.

Un método inmunológico ha sido el acoplamiento de anticuerpos antiooquiste con fosfatasa alcalina sobre NPs de oro para detectar a Cryptosporidium parvum (Benelli, 2018). Asimismo, NPs de silicio fluorescentes con capacidad para unirse a la proteína $\mathrm{A}$ y un anticuerpo monoclonal antiamiba fueron usadas para detectar trofozoítos de E. histolytica por medio de microscopía de fluorescencia indirecta (Hemadi et al., 2015). En otros estudios, NPs de oro funcionalizadas se conjugaron con cuatro sondas de oligonucleótidos para detectar el DNA circular del cinetoplástido de Leishmania spp. en muestras clínicas, mientras que otra alternativa para el mismo parásito ha sido la detección de productos de PCR usando NPs de oro y flujo lateral de 
ácidos nucleicos (Akbari et al., 2017). Recientemente, se ha reportado un biosensor para E. histolytica que detecta un amplicón marcado con digoxigenina conjugado a NPs de oro (Foo et al., 2017). En el ámbito de la prevención, las vacunas también son prometedoras pues están en desarrollo sistemas nanométricos para Plasmodium y Leishmania que actúan como adyuvantes o acarreadores de antígenos (Benelli, 2018).

Todavía no es posible evaluar la utilidad real de todas estas nuevas tecnologías usando a las NPs, pero se espera comparar en el futuro con los métodos de referencia inmunológicos o moleculares ya existentes. Las ventajas y utilidad de usar NPs acopladas a otras moléculas y poder usarlas para, a su vez, detectar proteínas o ácidos nucleicos, en vez de anticuerpos, fluoróforos, etc., es que se aumenta la sensibilidad de la detección, se reduce el costo y se facilita su uso en diferentes condiciones. A pesar de que recientemente se han desarrollado diferentes metodologías para la evaluación antimicrobiana de NPs y su interacción con microrganismos, es importante considerar todos los procesos celulares involucrados y sus posibles efectos tóxicos al interactuar con organismos vivos, por lo que el estudio de la toxicidad de los nanomateriales representa un importante aporte científico (Sharifi et al., 2012). La regulación y cuestiones bioéticas sobre el uso de nanomateriales son temas de especial relevancia que requieren una revisión exhaustiva por especialistas en dichas áreas.

\section{Evaluación de la actividad antimicrobiana de nanocompuestos}

Se han establecido una variedad de técnicas de microbiología clásica para evaluar la viabilidad de las bacterias y la eficacia de las NPs como agentes antibacterianos. Sin embargo, para la evaluación de la actividad antimicrobiana de diversos nanomateriales que se pretenden introducir al mercado, se siguen diversos métodos y procedimiento de acuerdo con estándares internacionales tales como: International Organization for Standardization (ISO), American Association of Textile Chemists and Colorists (AATCC), American Standard and Testing of Materials (ASTM), Japanese Industrial Standards (JIS), entre otros. Dependiendo de la naturaleza del compuesto nanoestructurado y de su potencial aplicación, se siguen protocolos para validar su actividad antimicrobiana. De esta manera, existen protocolos para plásticos, metales (JIS z 2801) y telas (AATCC-100-2012/ISO105-C06) entre otros, para lo cual se emplean bacterias u hongos de referencia (ATCC). La principal diferencia entre los protocolos es el inóculo del microrganismo, temperatura, tiempo de incubación y medio de cultivo. 


\section{Referencias}

Ae J., Huh, Young, J., Kwon (2011). Nanoantibiotics: A new paradigm for treating infectious diseases using nanomaterials in the antibiotics resistant era. Journal of Controlled Release, 156: 128-45. https://doi.org/10.1016/j.jconrel.2011.07.002

Aderibigbe, B. A. (2017). Metal-based nanoparticles for the treatment of infectious diseases. Molecules, 22: 1370. https://doi.org/10.3390/molecules22081370

Akbari, M., Oryan, A., Hatam, G. (2017). Application of nanotechnology in treatment of leishmaniasis: A review". Acta Tropica, 17: 86-90. https://doi.org/10.1016/j.actatropica.2017.04.029

Auffan, M., Rose, J., Bottero, J.-Y., Lowry, G. V., Jolivet, J.-P., Wiesner, M. R. (2009). Towards a definition of inorganic nanoparticles from an environmental, health and safety perspective. Nat. Nanotechnol., 4: 634-641.

https://doi.org/10.1038/nnano.2009.242

Benelli, G. (2018). Gold nanoparticles - against parasites and insect vectors. Acta Tropica 178: 73-80. https://doi.org/10.1016/j.actatropica.2017.10.021

Borase, H. P., Patil, C. D., Sauter, I. P., Rott, M. B., Patil, S. V. (2013). Amoebicidal activity of phytosynthesized silver nanoparticles and their in vitro cytotoxicity to human cells. FEMS Microbiol Lett., 345: 127-131.

https://doi.org/10.1111/1574-6968.12195

Chiang, W. L., Lin, T. T., Sureshbabu, R., Chia, W. T., Hsiao, H. C., Liu, H. Y., Yang, C. M., Sung, H. W. (2015). A rapid drug release system with a NIR light-activated molecular switch for dual-modality photothermal/antibiotic treatments of subcutaneous abscesses. J. Control Release, 10(99): 53-62.

https://doi.org/10.1016/j.jconrel.2014.12.011

Das, S. K., Das, A. R., Guha, A. K. (2009). Gold nanoparticles: Microbial synthesis and application in water hygiene management. Langmuir, 25: 8192-8199. https://doi.org/10.1021/la900585p

Dresselhaus, M. S., Jorio, A., Hofmann, M., Dresselhaus, G., Saito, R. (2010). Perspectives on carbon nanotubes and graphene Raman spectroscopy. Nano Lett., 10: 751-58. https://doi.org/10.1021/nl904286r

Elías A. L., Carrero-Sánchez, J. C., Terrones, H., Endo, M, Laclette, J. P., Terrones, M. (2007). Viability studies of pure carbon- and nitrogen-doped nanotubes with Entamoeba histolytica: From amoebicidal to biocompatible structures. Small, 3: 1723-1729. https://doi.org/10.1002/smll.200700331

España-Sánchez, B. L., Avila-Orta, C. A., Padilla-Vaca, F., Neira-Velazquez, M. G., González-Morones, P., Rodríguez-González, J.A., Hernández-Hernández, E., Rangel-Serrano, A., Díaz-Barriga E., Yate, L., Ziolo, R. F. (2014). Enhanced antibacterial activity of melt processed poly(propylene) Ag and cu nanocomposites by argon plasma treatment. Plasma Process. Polym., 11, 353-365.

https://doi.org/10.1002/ppap.201300152

España-Sánchez, B. L., Avila-Orta, C. A., Padilla-Vaca, F., Barriga-Castro, E. D., Soriano-Corral, F., González-Morones, P., Ramírez-Wong, D., Luna-Bárcenas, G. (2017). Early stages of antibacterial damage of metallic nanoparticles by 
Mundo Nano | ARTículos | www.mundonano.unam.mx

11(21), 15-28, julio-diciembre 2018 | http://dx.doi.org/10.22201/ceiich.24485691e.2018.21.62591

F. Padilla-Vaca, C. L. Mendoza-Macias, B.Franco, F. Anaya-Velázquez, P. Ponce-Noyola, A. Flores-Martínez

TEM and STEM-HAADF. Current Nanoscience, 13: 1-8.

https://doi.org/10.2174/2468187307666170906150731

Fedlheim, D. L., Foss, C. A. (2001). Metal nanoparticles: Synthesis, characterization, and applications. Nueva York, Basilea: CRC Press, $352 \mathrm{pp}$.

Foo, P. C., Chan, Y. Y., Mohamed, M., Wong, W. K., Nurul Najian, A. B., Lim, B. H. (2017). Development of a thermostabilised triplex LAMP assay with dryreagent four target lateral flow dipstick for detection of Entamoeba histolytica and non-pathogenic Entamoeba spp. Anal Chim Acta, 966: 71-80.

https://doi.org/10.1016/j.aca.2017.02.019

González-Vargas N. C., Mendoza-Macías C. L., Medina-Navarro L. G., Rangel-Serrano A., Padilla-Vaca L. F. (2017). Actividad antibacteriana de nanopartículas metálicas sobre bacterias resistentes a antibióticos convencionales. Jóvenes en la ciencia. Revista de divulgación científica, 3(2): 913-917. Verano de la Investigación Científica, Guanajuato, México.

Havlickova, B., Czaika, V. A., Friedrich, M. (2008). Epidemiological trends in skin mycoses worldwide. Mycoses, 51: 2-15.

https://doi.org/10.1111/j.1439-0507.2008.01606.x

Hamer D. H. (1986) Metallothionein. Annu. Rev. Biochem. 55: 913-951.

https://doi.org/10.1146/annurev.bi.55.070186.004405

Hemadi, A., Ekrami, A., Oormazdi, H., Meamar, A. R., Akhlaghi, L., Samarbaf-Zadeh, A.R., Razmjou, E. (2015). Bioconjugated fluorescent silica nanoparticles for the rapid detection of Entamoeba histolytica. Acta Trop. 145: 26-30.

https://doi.org/10.1016/j.actatropica.2015.02.008

Horan, T., Gaynes, R., Martone, W., Jarvis, W., Graceemori, T. (1992). CDC definitions of nosocomial surgical site infections: A modification of CDC definitions of surgical wound infections. Am. J. Infect. Control, 20: 271-274.

https://doi.org/10.1016/S0196-6553(05)80201-9

Islas Robles, A. (2013) Estudio de la inducción de la resistencia a cobre en cepas del género Candida. Tesis DCNE, Universidad de Guanajuato.

Jutkina, J., Marathe, N.P., Flach, C.F., Larsson, D.G.J. (2017) Antibiotics and common antibacterial biocides stimulate horizontal transfer of resistance at low concentrations. Sci Total Environ. 4; 616-617: 172-178.

https://doi.org/10.1016/j.scitotenv.2017.10.312

Kim, M. H. (2016). Nanoparticle-based therapies for wound biofilm infection: Opportunities and challenges. IEEE Trans Nanobioscience, 15(3): 294-304. https://doi.org/10.1109/TNB.2016.2527600

Lerch K. (1980). Copper metallothionein, a copper-binding protein from Neurospora crassa. Nature, 284: 368-370. https://doi.org/10.1038/284368a0

Lok C., Chen, R., He, Q., Yu, W., Sun, H., Tam, P., Chiu, J., Che, C. (2006). Proteomic analysis of the mode of antibacterial action of silver nanoparticles. J. Proteome Res., 5: 916-924. https://doi.org/10.1021/pr0504079

Luna-Hernández, E., Cruz-Soto, M. E., Padilla-Vaca, F., Mauricio-Sánchez, R. A., Ramirez-Wong, D., Muñoz, R., Granados-López, L., Ovalle-Flores, L. R., Menchaca-Arredondo, J. R., Hernández-Rangel, A., Prokhorov E., García-Rivas, J. L., Es- 
paña-Sánchez, B. L., Luna-Bárcenas, G. (2017). Combined antibacterial/tissue regeneration response in thermalburns promoted by functional chitosan/silver nanocomposites. International Journal of Biological Macromolecules, 105(Pt 1): 1241-1249. https://doi.org/10.1016/j.ijbiomac.2017.07.159

Melzer, M., Eykyn, S. J., Gransden, W. R., Chinn, S. (2003) Is methicillin-resistant Staphylococcus aureus more virulent than methicillin-susceptible $S$. aureus? A comparative cohort study of British patients with nosocomial infection and bacteremia. Clin. Infect., 37: 1453-1460. https://doi.org/10.1086/379321

Morones, J. R., Elechiguerra, J. L., Camacho, A., Holt, K., Kouri, J. B., Ramírez, J. T., Yacaman, M. J. (2005). The bactericidal effect of silver nanoparticles. Nanotechnology, 16: 2346-2353. https://doi.org/10.1088/0957-4484/16/10/059

Mulvaney, P. (2015). Nanoscience vs nanotechnology-defining the field. ACS Nano, 24, 9(3): 2215-7. https://doi.org/10.1021/acsnano.5b01418

Pham D. C., Nguyen T. H., Ngoc U. T., Le N. T., Tran T. V., Nguyen D. H. (2018). Preparation, characterization and antifungal properties of chitosan-silver nanoparticles synergize fungicide against Pyricularia oryzae. J Nanosci Nanotechnol., 1, 18(8): 5299-5305. https://doi.org/10.1166/jnn.2018.15400

Ponte-Sucre, A., Gamarro, F., Dujardin, J. C., Barrett, M. P., López-Vélez, R., GarcíaHernández, R., Pountain, A. W., Mwenechanya, R., Papadopoulou, B. (2017). Drug resistance and treatment failure in leishmaniasis: A 21st century challenge. PLoS Negl Trop Dis., 14, 11(12). https://doi.org/10.1371/journal.pntd.0006052

Rónavári, A., Igaz N., Gopisetty, M. K., Szerencsés, B., Kovács, D., Papp C., Vágvölgyi, C., Boros, I. M., Kónya, Z., Kiricsi, M., Pfeiffer, I. (2018). Biosynthesized silver and gold nanoparticles are potent antimycotics against opportunistic pathogenic yeasts and dermatophytes. Int J Nanomedicine, 1(13): 695-703.

https://doi.org/10.2147/IJN.S152010

Saad, H. A., Soliman, M. I., Azzam, A. M., Mostafa, B. (2015). Antiparasitic activity of silver and copper oxide nanoparticles against Entamoeba histolytica and Cryptosporidium parvum cysts. J Egypt Soc Parasitol, 45: 593-602. https://doi.org/10.12816/0017920

Said, D. E., Elsamad, L. M., Gohar, Y. M. (2012). Validity of silver, chitosan, and curcumin nanoparticles as anti-Giardia agents. Parasitol Res., 111: 545-554. https://doi.org/10.1007/s00436-012-2866-1

Scalise, M. L., Arrúa, E. C., Rial, M. S., Esteva, M. I., Salomon, C. J. (2016). Promising efficacy of benznidazole nanoparticles in acute Trypanosoma cruzi Murine model: in-vitro and in-vivo studies. Am J Trop Med Hyg., 95: 388-393. https://doi.org/10.4269/ajtmh.15-0889

Seil, J. T., Webster, T. J. (2012). Antimicrobial applications of nanotechnology: Methods and literature. Int. J. Nanomedicine, 7: 2767-2781.

https://doi.org/10.2147/IJN.S24805

Sharifi, S., Behzadi, S., Laurent, S., Forrest, M. L., Stroeve, P., Mahmoudi, M. (2012).

Toxicity of nanomaterials. Chemical Society Reviews, 41: 2323-2343.

https://doi.org/10.1039/C1CS15188F

Short, E. E., Caminade, C., Thomas, B. N. (2017). Climate change contribution to the 
emergence or re-emergence of parasitic diseases. Infect Dis: Research and treatment, 10: 1-7. https://doi.org/10.1177/1178633617732296

Shrivastava, S., Bera, T., Roy, A., Singh, G., Ramachandrarao, P., Dash, D. (2007). Characterization of enhanced antibacterial effects of novel silver nanoparticles. Nanotechnology, 18: 225103.

Tedesco, S., Doyle, H., Blasco, J., Redmond, G., Sheehan, D. (2010). Oxidative stress and toxicity of gold nanoparticles in Mytilus edulis. Aquat. Toxicol., 100: 178-186. https://doi.org/10.1016/j.aquatox.2010.03.001

Vimbela, G. V., Ngo, S. M., Fraze, C., Yang, L., Stout, D. A. (2017). Antibacterial properties and toxicity from metallic nanomaterials. Int. J. Nanomedicine, 24(12): 3941-3965. https://doi.org/10.2147/IJN.S134526

Wang, L., He, H., Yu, Y., Sun, L., Liu, S., Zhang, C. et al. (2014). Morphology-dependent bactericidal activities of $\mathrm{Ag} / \mathrm{CeO}_{2}$ catalysts against Escherichia coli. J. Inorg. Biochem., 135: 45-53.

Wu, X., Kwon, S. J., Kim, J., Kane, R. S, Dordick, J. S. (2017). Biocatalytic nanocomposites for combating bacterial pathogens. Annu. Rev. Chem. Biomol. Eng., 7(8): 87-113. https://doi.org/10.1146/annurev-chembioeng-060816-101612

Zhang, L., Pornpattananangkul, D., Hu, M., Huang, C. (2010). Development of nanoparticles for antimicrobial drug delivery. Current Medicinal Chemistry, 17(6): 585594. 